\title{
Hemorheological factors can be informative in comparing treatment possibilities of abdominal compartment syndrome
}

\author{
Norbert Nemeth $^{\mathrm{a}, *}$, Katalin Peto ${ }^{\mathrm{a}}$, Adam Deak ${ }^{\mathrm{a}}$, Viktoria Sogor ${ }^{\mathrm{a}}$, Gabor Varga ${ }^{\mathrm{a}}$, \\ Bence Tanczos $^{\mathrm{a}}$, Klaudia Balog ${ }^{\mathrm{b}}$, Adrienn Csiszko ${ }^{\mathrm{b}}$, Zoltan Godo $^{\mathrm{c}}$ \\ and Zsolt Szentkereszty \\ ${ }^{a}$ Department of Operative Techniques \& Surgical Research, Faculty of Medicine, \\ University of Debrecen, Hungary \\ ${ }^{\mathrm{b}}$ Institute of Surgery, Faculty of Medicine, University of Debrecen, Hungary \\ ${ }^{\mathrm{c}}$ Department of Information Technology, Faculty of Informatics, University of Debrecen, Hungary
}

\begin{abstract}
.
BACKGROUND: Abdominal compartment syndrome (ACS) is a life-threatening condition, of which pathomechanism hasn't been completely clarified, yet. Furthermore, surgical therapy still needs optimization.

OBJECTIVE: We aimed to investigate microcirculatory and micro-rheological alterations in ACS, using various temporary abdominal closure methods, including three settings of vacuum-assisted closure technique (negative pressure wound therapy, NPWT).

METHODS: On anesthetized pigs, by intraabdominally placed and filled-up silicone bags, intraabdominal pressure at $30 \mathrm{mmHg}$ was maintained for 3 hours, and afterwards, decompressive laparotomy happened. In different experimental groups Bogota-bag or Vivano-sets were applied $(-50,-100,-150 \mathrm{mmHg})$ for 2 hours. Pressure monitoring was done by implanted sensors, hemorheological parameters were determined, and laser Doppler flowmetry tests were performed on the surface of intraabdominal organs.

RESULTS: Treatment with Bogota-bag and $-150 \mathrm{mmHg}$ vacuum increased erythrocyte aggregation, while deformability declined. Blood viscosity increased after treatment with $-150 \mathrm{mmHg}$ vacuum. The microcirculatory parameters of the NPWT groups were better in case of the small intestine.

CONCLUSIONS: ACS resulted in impairment of macro- and micro-rheological parameters and abdominal organs' microcirculation. All of the used techniques improved the results, however, applying Bogota-bag or $-150 \mathrm{mmHg}$ vacuum set showed worse microcirculatory and micro-rheological data than the settings at -100 or $-50 \mathrm{mmHg}$.
\end{abstract}

Keywords: Abdominal compartment syndrome, negative pressure wound therapy, microcirculation, hemorheology

\section{Introduction}

Intra-abdominal hypertension (IAH) is known as a serious complication of critically ill patients. Without appropriate treatment abdominal compartment syndrome (ACS) may develop, that is a lifethreatening condition even nowadays $[1,10,18]$. The definition of ACS, based on the 2013 guidelines of the World Society of the Abdominal Compartment Syndrome (WSACS) reads as follows: "ACS is defined as a sustained IAP $>20 \mathrm{mmHg}$ (with or without an APP $<60 \mathrm{mmHg}$ ) that is associated with

\footnotetext{
*Corresponding author: Norbert Nemeth, MD, PhD, Department of Operative Techniques and Surgical Research, Institute of Surgery, Faculty of Medicine, University of Debrecen, H-4032 Debrecen, Nagyerdeikrt 98, Hungary. Tel./Fax: +36 52416 915; E-mail: nemeth@med.unideb.hu.
} 
new organ dysfunction/failure". The APP is defined as the mean arterial pressure (MAP) minus the IAP $[11,15,16]$.

Normal value of intra-abdominal pressure (IAP) does not exceed 5-7 mmHg. Several primary (e.g., blunt/penetrating trauma, ruptured abdominal aorta aneurysm, mechanical intestinal obstruction, postoperative bleedings, etc.) and secondary conditions (e.g., severe intra-abdominal infection, ascites, pancreatitis, ileus, sepsis, major burns, etc.) can cause intra-abdominal hypertension [5, 21, 22, 26, 28]. There is also an entity known as recurrent (or tertier) ACS. Regardless of the inciting event, all lead to a capillary leak syndrome and bowel wall edema, which leads to increased intra-abdominal pressure and ultimately organ dysfunction occurs. The primer conditions needs urgent surgical or interventional radiological treatment, while in secondary conditions conservative therapies might be successful, but in case of their ineffectiveness surgical intervention is needed $[6,15]$. The scale is colourful and the sequence between the intra-abdominal hypertension (IAH) and abdominal compartment syndrome (ACS) is a continuous line without sharp border. The mortality of ACS is very high (even $80 \%$ ) $[11,15,16,24]$. Despite the very high mortality rate of the syndrome, little is known of the extent and influencing factors of these microcirculatory changes.

The intra-abdominal hypertension might occur suddenly or progressively according to the etiology by the magnitude for grades are known (grade I: $12-15 \mathrm{mmHg}$, grade II: $16-20 \mathrm{mmHg}$, grade III: 21-25 mmHg, grade IV: $>25 \mathrm{mmHg}$ ). Abdominal compartment syndrome is defined as a sustained IAP over $20 \mathrm{mmHg}$ (IAH grade III-IV), when the abdominal perfusion pressure decreases and the intra-abdominal hypertension is associated with any organ dysfunction [15, 24]. In development of organ dysfunction besides the decreased perfusion pressure and hemodynamical alterations, supposedly hemorheological and microcirculatory deterioration also might play a role $[3,14,20]$.

However, hemorheological relations of the abdominal compartment syndrome have not been elucidated yet. The rheological parameters of the circulating blood are important factors in macro- and microcirculation [7]. Micro-rheological parameters, such as red blood cell deformability and red blood cell aggregation are highly important ones, since impaired deformability and enhanced aggregation lead to microcirculatory deterioration $[3,7,19]$. Therefore conducting experiments on these parameters is of upmost importance, we believe, especially in surgical pathophysiology research. Decrease in blood due to any reasons leads to a circulatory insufficiency and disturbance in tissue perfusion. This will cause further negative changes in the rheological parameters of the blood via local metabolic changes and tissue damage associated acute phase reactions, resulting in the elevation of blood-viscosity which, in turn, closing the vicious circle, would lead to further decrease in perfusion and disturbances of the circulation [3].

To prevent and to treat perfusion disturbances, a surgical approach is needed. The aim of the surgical intervention is decompression, and, if possible, the elimination of the etiology. Above $20 \mathrm{mmHg}$ of IAP and/or organic failure decompressive laparotomy is needed (e.g., median, transverse transrectal, bilateral subcostal). Partial solution is the subcutan linea alba fasciotomy. Afterwards temporary abdominal closure (TAC) and further treatment methods are necessary to be applied, such as fascia closing by retention stitches, closing the cutis by clips (deeper layers opened), zipper systems, Wittmann-patch, or using various surgical meshes (even combined), Bogota-bag, or Vacuum Assisted Closure (VAC) Negative Pressure Wound Therapy (NPWT) $[6,8,12,15,16,27]$. The optimal value of negative pressure during the NPWT/VAC, however, is not yet supported with enough objective data when referring to the abdominal compartment syndrome, and there is only a very limited amount of available research in literature [e.g., 4].

The objective of this study was the comparative analysis of surgical treatment methods of the abdominal compartment syndrome (Bogota-bag, NPWT at $-50,-100$, or $-150 \mathrm{mmHg}$ settings) investigating microcirculation of selected intra-abdominal organs, and the influencing micro-rheological parameters in a porcine model. We hypothesized that abdominal compartment syndrome may cause 
micro-rheological alterations, and the various NPWT settings show the effectiveness in different manner on reducing microcirculatory disturbances.

\section{Materials and methods}

\subsection{Experimental animals, operative techniques and sampling protocol}

The animal experiment parts were approved and registered by the University of Debrecen Committee of Animal Welfare (permission Nr.: 13/2014/UDCAW), in accordance with national and EU regulations (the Hungarian Animal Protection Act (Law XVIII/1998) and the Edict 63/2010).

Twenty-six female juvenile Hungahib $(17.52 \pm 1.75 \mathrm{~kg})$ were anaesthetized $(15 \mathrm{mg} / \mathrm{kg}$ Ketamin + $1 \mathrm{mg} / \mathrm{kg}$ Xylazine, maintenance: half-dose combination, in case of necessity). Tracheostomy inferior was performed for assisted ventilation and the left external jugular vein and the left femoral artery were prepared and cannulated for hemodynamic measurement, blood samplings and volume therapy. Via epicystostomy a catheter was introduced into the urinary bladder for determining hourly urine output. A 2-3-cm incision was maid above the symphysis a sterile elastic silicon bag was placed into the abdominal cavity, and it was tilled up with body temperature physiological solution till the intraabdominal pressure reached $30 \mathrm{mmHg}$. This condition was maintained for 3 hours. By a connecting pressure monitor the intra-abdominal pressure was regularly checked. Following the 3-hour intraabdominal hypertension face the silicon bag was drained and removed, and decompressive median laparotomy was performed.

According to the experimental groups different abdominal closure and treatment methods were applied. Bogota-bag was sutured $(n=6)$, or Vivano-abdominal sets (negative pressure wound therapy, NPWT) were applied using various vacuum settings: $-50 \mathrm{mmHg}(n=7),-100 \mathrm{mmHg}(n=7)$, or $-150 \mathrm{mmHg}(n=6)$, respectively. After 2 hours, the Bogota-bag or the Vivano-abdominal sets were removed.

Blood samplings (6-8 $\mathrm{ml}$ each, $\mathrm{K}_{3}$-EDTA) via the cannulated vein were performed before and in the 1st, 2nd and 3rd hour of the IAH period, and in the 1st and 2nd hour of the treatment phase as well. Equal volume of physiological saline solution was replaced intravenously.

Microcirculatory measurements were carried out just after performing the decompressive laparotomy and after the 2-hour treatment period.

Before using the temporary abdominal closure technique or the Vivano-abdominal set, special pressure sensors were placed into the abdominal cavity at various points. The multichannel pressure monitor device was a custom-made development by Zoltan Godo (Department of Information Technology, Faculty of Informatics, University of Debrecen).

\subsection{Laboratory methods}

Hematological parameters were tested by a Sysmex F-800 semi-automated microcell counter (TOA Medical Electronics Co., Ltd., Japan).

Blood and plasma viscosity determine by a capillary viscosimeter (Hevimet-40, Hemorex Ltd., Hungary). The whole blood viscosity values were corrected for $40 \%$ hematocrit, using the Mátraiformula: $\mathrm{WBV}_{40 \%} / \mathrm{PV}=\left(\mathrm{WBV}_{\mathrm{Hct}} / \mathrm{PV}\right)^{(40 \% / \mathrm{Hct})}$, where $\mathrm{WBV}_{40 \%}$ : corrected for $40 \% \mathrm{Hct}$; $\mathrm{WBV}_{\mathrm{Hct}}$ : whole blood viscosity measured at the native Hct; PV: plasma viscosity; Hct: actual hematocrit value [\%] of the sample [23].

For determining red blood cell aggregation we used light-transmittance and light-reflectance methods. A Myrenne MA-1 erythrocyte aggregometer (Myrenne GmbH, Germany) was used for 
determining aggregation index values $\mathrm{M}$ (at shear rate of $0 \mathrm{~s}^{-1}$ ) and $\mathrm{M} 1$ (at shear rate of $3 \mathrm{~s}^{-1}$ ) at 5 or 10 seconds after disaggregation. The indices (M $5 \mathrm{~s}$, M1 $5 \mathrm{~s}, \mathrm{M} 10 \mathrm{~s}, \mathrm{M} 110 \mathrm{~s}$ ) increase with enhanced red blood cell aggregation [9]. By the LoRRca syllectometry the following parameters were determined: amplitude (Amp [au]), aggregation index (AI [\%]) and the aggregation half-time $\left(\mathrm{t}_{1 / 2}[\mathrm{~s}]\right)[9]$.

Red blood cell deformability was determined by LoRRca MaxSis Osmoscan rotational ektacytometer (Mechatronics BV, The Netherlands), in which the cells' elongation index (EI) was tested in the function of shear stress (SS [Pa]) [9]. Measurements were carried out at $37^{\circ} \mathrm{C}$. Polyvinylpyrrolidone (PVP) phosphate buffered saline (PBS) solution was used as high-viscosity suspending media (PVP: $360 \mathrm{kDa}$, Sigma-Aldrich Co. USA; PVP-PBS solution viscosity $=30.83 \mathrm{mPas}$, osmolality $=298 \mathrm{mOsmol} / \mathrm{kg}$, $\mathrm{pH}=7.2$ ). For the comparison of the EI-SS curves the Lineweaver-Burk analysis was applied, and the ratio of maximal elongation index $\left(\mathrm{EI}_{\max }\right)$ and the shear stress value at half $\mathrm{EI}_{\max }\left(\mathrm{SS}_{1 / 2}[\mathrm{~Pa}]\right)$ was used [2].

In osmotic gradient ektacytometry (osmoscan) test the elongation index values were continuously determined at constant shear stress (30 Pa), while the osmolality was changing $(0-500 \mathrm{mOsmol} / \mathrm{kg})$ [9]. Among the device-given parameters we analyzed the minimal elongation index values measured at low-osmotic environment (minimal EI), the maximal elongation index values (maximal EI, not equal to $\mathrm{EI}_{\max }$ ), the belonging osmolality values ( $\mathrm{minO}$ and $\operatorname{maxO}$ ), and the area under the individual elongation index-osmolality curves (AUC).

\subsection{Microcirculatory measurements}

After decompressive laparotomy and at the end of the 2-hour treatment period, microcirculation was monitored by laser Doppler technique (LD-01 Laser Doppler Flowmeter, Experimetria Ltd., Hungary), using a standard pencil probe (Oxford Optronix Ltd., UK). The device determines blood flux unit (BFU [au]) based on the number of moving red blood cells and their mean velocity in the tested tissue volume $\left(1-1.5 \mathrm{~mm}^{3}\right)$ [17]. the probe was gently placed on the surface of the greater omentum $(1-2 \mathrm{~cm}$ from the right angle), on the middle region of the right liver lobe, on the head of the pancreas, on the antimesenterial surface of a jejunum loop and on the anterior middle surface of the right kidney, consecutively. The signal was recorded by S.P.E.L. Advanced Kymograph software (Experimetria Ltd., Hungary) at $1 \mathrm{kHz}$ sampling rate for $30-60$ seconds. During off-line data analysis the average value of a noise-free 10-second long representative section of each recorded graph was calculated.

\subsection{Statistical analysis}

Data are presented as means \pm standard deviation (S.D.). One way and repeated measures ANOVA tests were used for intra- and inter-group comparisons (Bonferroni/Dunn methods). For simple comparison of inter-group differences at single time points, $t$-test/Mann-Whitney rank sum tests were applied as well, depending on the normality of data distribution. A $p<0.05$ value was considered statistically significant.

\section{Results}

\subsection{General observations}

Mean arterial pressure decreased, central venous pressure increased during the intra-abdominal hypertension (IAH) period (data not shown). The $30 \mathrm{mmHg}$ could be maintained well during the 
3-hour period. Diuresis (hourly urine output) decreased. By definition, the abdominal compartment syndrome occurred. Exitus happened in all groups (one/each) in the 2nd and 3rd hours of the increased intra-abdominal pressure period.

\subsection{Hematological parameters}

White blood cell count increased in all groups gradually over the intra-abdominal hypertension period, and showed further rise mostly in -50 and $-100 \mathrm{mmHg}$ groups by the end of the experiments (I. Bogota-group base: $12.45 \pm 3.27$, end of IAH: $16.3 \pm 6.63$, end of experiment $18.07 \pm 7.3610^{9} / \mathrm{L}$; II. $-50 \mathrm{mmHg}$ group base: $14.91 \pm 5.88$, end of IAH: $23.61 \pm 5.88$, end of experiment: $23.72 \pm 8.62$ $10^{9} / \mathrm{L}$; III. $-100 \mathrm{mmHg}$ group base: $14.89 \pm 2.91$, end of IAH: $24.01 \pm 10.15$, end of experiment: $28.6 \pm 11.2810^{9} / \mathrm{L}$; IV. $-150 \mathrm{mmHg}$ group base: $15.13 \pm 4.69$, end of IAH $24.94 \pm 10.82$, end of experiment: $\left.19.43 \pm 7.2810^{9} / \mathrm{L}\right)$.

Platelet count showed a moderate decrease over the IAH then slightly increased without significant difference. Qualitative red blood cell parameters did not show important changes. Hematocrit values decreased over of experiment without significant difference (I. Bogota-group base: $40.04 \pm 9.85$, end of IAH: $39.97 \pm 4.63$, end of experiment $40.3 \pm 4.34 \%$; II. $-50 \mathrm{mmHg}$ group base: $35.55 \pm 3.67$, end of IAH: $41.04 \pm 4.83$, end of experiment: $38.53 \pm 4.82 \%$; III. $-100 \mathrm{mmHg}$ group base: $35.75 \pm 1.87$, end of IAH: $41.48 \pm 4.47$, end of experiment: $38.28 \pm 4.65 \%$; IV. $-150 \mathrm{mmHg}$ group base: $38.07 \pm 6.02$, end of IAH $38.61 \pm 6.03$, end of experiment: $34.85 \pm 3.95 \%$ ).

\subsection{Blood and plasma viscosity}

Whole blood viscosity at $90 \mathrm{~s}^{-1}$ shear rate slightly increased in Bogota and $-50 \mathrm{mmHg}$ groups and moderately decreased in -100 and $-150 \mathrm{mmHg}$ groups. The corrected values for $40 \%$ hematocrit showed a relative increase in $-150 \mathrm{mmHg}$ groups, while the values of -50 and $-150 \mathrm{mmHg}$ groups were lower compared to the Bogota-group (Table 1).

\subsection{Red blood cell aggregation}

The red blood cell aggregation index values gradually increased over the 3-hour period of intraabdominal hypertension. By the end of the treatment period the lowest values were found in the $-100 \mathrm{mmHg}$ group (Table 1, Fig. 1A).

Aggregation index \% (AI\%) showed a minimal increase over the IAH then it decreased by the end of the experiment in all groups. Amplitude increased by the end of the experiment without significant difference between groups. $\mathrm{T}_{1 / 2}$ values increased after the IAH period resulting in the highest values in the -100 and $-150 \mathrm{mmHg}$ groups (Table 1 ).

\subsection{Red blood cell deformability (normal and osmotic gradient ektacytometry)}

Red blood cell deformability did not show important changes during the intra-abdominal hypertension period. Significantly lower values were found in Bogota-group and $-150 \mathrm{mmHg}$ group in the 1st and 2nd hours of the treatment period (Fig. 1B).

Osmotic gradient ektacytometry parameter did not show important changes, however the AUC continuously decreased over the experiment (I. Bogota-group base: $136.55 \pm 3.73$, end of IAH: $129.91 \pm 3.9$, end of experiment $130.4 \pm 3.41$; II. $-50 \mathrm{mmHg}$ group base: $135.67 \pm 4.21$, end of IAH: $126.6 \pm 11.34$, end of experiment: $129.84 \pm 5.73$; III. $-100 \mathrm{mmHg}$ group base: $139.55 \pm 4.67$, end of IAH: $128.55 \pm 3.44$, end of experiment: $129.23 \pm 3.91$; IV. $-150 \mathrm{mmHg}$ group base: $134.51 \pm 6.43$, 
Table 1

Whole blood viscosity values corrected for $40 \%$ hematocrit $\left(\mathrm{WBV}_{40 \%}\right)$, red blood cell aggregation parameters determined by Myrenne aggregometer (M $5 \mathrm{~s}, \mathrm{M} 10 \mathrm{~s}, \mathrm{M} 15 \mathrm{~s}$ and M1 $10 \mathrm{~s}$ indices) and the LoRRca (AI\%, Amp, $\mathrm{t}_{1 / 2}$ ) during the 3-hour 30-mmHg intra-abdominal hypertension period, and after decompression, during the consecutive 2-hour application of various abdominal closure techniques ("Treatment": Bogota-bag, NPWT at $-50,-100$ and $-150 \mathrm{mmHg}$ )

\begin{tabular}{|c|c|c|c|c|c|c|c|}
\hline \multirow[t]{2}{*}{ Variable } & \multirow[t]{2}{*}{ Group } & \multirow[t]{2}{*}{ Base } & \multicolumn{3}{|c|}{ Intra-abdominal hypertension phase ( 3 hours) } & \multicolumn{2}{|c|}{ Treatment phase ( 2 hours) } \\
\hline & & & $1 \mathrm{~h}$ & $2 \mathrm{~h}$ & $3 \mathrm{~h}$ & $4 \mathrm{~h}$ & $5 \mathrm{~h}$ \\
\hline \multirow[t]{4}{*}{$\begin{array}{r}\mathrm{WBV}_{40 \%} \\
{[\mathrm{mPas}]}\end{array}$} & Bogota & $3.19 \pm 0.28$ & - & - & $\begin{array}{c}2.99 \pm 0.2 \\
(0.91 \pm 0.07)\end{array}$ & - & $\begin{array}{c}3.2 \pm 0.65 \\
(1.04 \pm 0.11)\end{array}$ \\
\hline & $-50 \mathrm{Hgmm}$ & $3.3 \pm 0.016$ & - & - & $\begin{array}{r}3.2 \pm 0.13 \\
(0.97 \pm 0.1)\end{array}$ & - & $\begin{array}{c}3.09 \pm 0.21 \\
(0.95 \pm 0.06)\end{array}$ \\
\hline & -100 Hgmm & $3.54 \pm 1.06$ & - & - & $\begin{aligned} 3.44 & \pm 0.6 \\
(1 & \pm 0.25)\end{aligned}$ & & $\begin{array}{c}3.29 \pm 0.48 \\
(0.95 \pm 0.21)\end{array}$ \\
\hline & $-150 \mathrm{Hgmm}$ & $2.99 \pm 0.65$ & - & - & $\begin{array}{c}2.98 \pm 0.41 \\
(1.05 \pm 0.33)\end{array}$ & & $\begin{array}{c}3.19 \pm 1.12 \\
(1.14 \pm 0.35)\end{array}$ \\
\hline \multirow[t]{4}{*}{ M $5 \mathrm{~s}$} & Bogota & $1.98 \pm 0.94$ & $\begin{array}{c}2.44 \pm 0.95 \\
(1.33 \pm 0.63)\end{array}$ & $\begin{array}{c}2.89 \pm 1.08 \\
(1.66 \pm 0.95)\end{array}$ & $\begin{array}{c}2.71 \pm 0.99 \\
(1.42 \pm 0.74)\end{array}$ & $\begin{array}{c}2.54 \pm 0.89 \\
(1.56 \pm 1.16)\end{array}$ & $\begin{array}{c}2.55 \pm 0.77 \\
(1.78 \pm 1.03)\end{array}$ \\
\hline & $-50 \mathrm{Hgmm}$ & $1.49 \pm 0.69$ & $\begin{array}{c}1.76 \pm 0.55 \\
(1.14 \pm 0.39)\end{array}$ & $\begin{array}{r}1.9 \pm 0.67 \\
(1.23 \pm 0.6)\end{array}$ & $\begin{array}{c}1.75 \pm 0.88 \\
(1.28 \pm 0.66)\end{array}$ & $\begin{array}{c}2.53 \pm 1.23^{*} \\
(1.67 \pm 0.75)\end{array}$ & $\begin{array}{c}2.6 \pm 0.78 \\
(1.96 \pm 0.99)\end{array}$ \\
\hline & -100 Hgmm & $1.58 \pm 0.84$ & $\begin{array}{c}2 \pm 0.6 \\
(1.01 \pm 0.35)\end{array}$ & $\begin{array}{l}1.54 \pm 0.76 \\
(0.9 \pm 0.62)\end{array}$ & $\begin{array}{c}2.07 \pm 1.44 \\
(0.78 \pm 0.29)\end{array}$ & $\begin{array}{l}1.86 \pm 0.64 \\
(1.1 \pm 0.48)\end{array}$ & $\begin{array}{c}1.71 \pm 0.72 \\
(0.84 \pm 0.37)\end{array}$ \\
\hline & $-150 \mathrm{Hgmm}$ & $2.04 \pm 0.88$ & $\begin{array}{c}2.57 \pm 0.92 \\
(1.27 \pm 0.35)\end{array}$ & $\begin{array}{c}2.75 \pm 0.97 \\
(1.91 \pm 0.83)\end{array}$ & $\begin{array}{c}3.12 \pm 0.63 \\
(2.01 \pm 1.41)\end{array}$ & $\begin{array}{c}2.23 \pm 0.66 \\
(1.42 \pm 0.99)\end{array}$ & $\begin{array}{r}2.47 \pm 0.82^{+} \\
(1.62 \pm 0.65)\end{array}$ \\
\hline \multirow[t]{4}{*}{ M $10 \mathrm{~s}$} & Bogota & $7.39 \pm 1.98$ & $\begin{array}{c}8.31 \pm 3.1 \\
(1.22 \pm 0.41)\end{array}$ & $\begin{array}{c}8.58 \pm 4.01 \\
(1.08 \pm 0.29)\end{array}$ & $\begin{array}{c}7.63 \pm 1.61 \\
(0.99 \pm 0.15)\end{array}$ & $\begin{array}{c}8.13 \pm 2.73 \\
(1.1 \pm 0.27)\end{array}$ & $\begin{array}{c}7.35 \pm 1.36 \\
(1.04 \pm 0.26)\end{array}$ \\
\hline & $-50 \mathrm{Hgmm}$ & $5.92 \pm 2.17$ & $\begin{array}{c}5.95 \pm 1.81 \\
(0.88 \pm 0.12)\end{array}$ & $\begin{array}{c}6.2 \pm 2.31 \\
(0.87 \pm 0.35)\end{array}$ & $\begin{array}{c}5.31 \pm 2.32 \\
(0.79 \pm 0.35)\end{array}$ & $\begin{array}{c}4.57 \pm 2.72 \\
(1.03 \pm 0.52)\end{array}$ & $\begin{array}{c}6.85 \pm 3.44 \\
(1.09 \pm 0.29)\end{array}$ \\
\hline & $-100 \mathrm{Hgmm}$ & $4.66 \pm 2.47$ & $\begin{array}{c}5.8 \pm 3.01 \\
(1.07 \pm 0.49)\end{array}$ & $\begin{array}{c}4.58 \pm 1.75 \\
(0.79 \pm 0.49)\end{array}$ & $\begin{array}{c}5.1 \pm 1.93 \\
(0.81 \pm 0.32)\end{array}$ & $\begin{array}{c}4.92 \pm 1.94 \\
(0.86 \pm 0.25)\end{array}$ & $\begin{array}{c}6.35 \pm 2.33 \\
(0.95 \pm 0.28)\end{array}$ \\
\hline & $-150 \mathrm{Hgmm}$ & $8.61 \pm 2.07$ & $\begin{array}{c}8.24 \pm 3.46 \\
(1.1 \pm 0.3)\end{array}$ & $\begin{array}{c}8.32 \pm 4.03 \\
(1.1 \pm 0.59)\end{array}$ & $\begin{array}{c}8.35 \pm 3.46 \\
(0.99 \pm 0.69)\end{array}$ & $\begin{array}{c}6.9 \pm 2.75 \\
(0.98 \pm 0.53)\end{array}$ & $\begin{array}{l}8.28 \pm 2.45 \\
(1.2 \pm 0.35)\end{array}$ \\
\hline \multirow[t]{4}{*}{ M1 $5 \mathrm{~s}$} & Bogota & $3.46 \pm 0.83$ & $\begin{array}{l}5.24 \pm 1.77 \\
(1.6 \pm 0.62)\end{array}$ & $\begin{array}{c}5.04 \pm 1.31 \\
(1.49 \pm 0.4)\end{array}$ & $\begin{array}{c}5.35 \pm 1.13 \\
(1.33 \pm 0.42)\end{array}$ & $\begin{array}{c}5.53 \pm 1.33 \\
(1.04 \pm 0.42)\end{array}$ & $\begin{array}{c}4.28 \pm 1.01 \\
(1.2 \pm 0.27)\end{array}$ \\
\hline & $-50 \mathrm{Hgmm}$ & $4.2 \pm 1.59$ & $\begin{array}{c}4.46 \pm 1.68 \\
(0.93 \pm 0.3)\end{array}$ & $\begin{array}{c}5.26 \pm 1.8 \\
(1.08 \pm 0.4)\end{array}$ & $\begin{array}{c}4.72 \pm 1.99 \\
(0.96 \pm 0.37)\end{array}$ & $\begin{array}{c}4.58 \pm 2.28 \\
(0.95 \pm 0.65)\end{array}$ & $\begin{array}{r}4.4 \pm 2.42 \\
(1.01 \pm 0.5)\end{array}$ \\
\hline & $-100 \mathrm{Hgmm}$ & $3.24 \pm 1.5$ & $\begin{array}{c}4.35 \pm 1.67 \\
(1.54 \pm 0.93)\end{array}$ & $\begin{array}{c}4.8 \pm 1.74 \\
(2.26 \pm 1.35)\end{array}$ & $\begin{array}{c}4.32 \pm 2.09 \\
(1.28 \pm 0.53)\end{array}$ & $\begin{array}{c}4.49 \pm 1.97 \\
(1.89 \pm 1.55)\end{array}$ & $\begin{array}{c}3.8 \pm 1.96 \\
(1.42 \pm 1.02)\end{array}$ \\
\hline & -150 Hgmm & $3.7 \pm 1.05$ & $\begin{array}{c}6.1 \pm 1.07 \\
(1.53 \pm 0.56)\end{array}$ & $\begin{array}{c}4.99 \pm 1.62 \\
(1.05 \pm 0.28)\end{array}$ & $\begin{array}{c}5.12 \pm 1.82 \\
(1.24 \pm 0.27)\end{array}$ & $\begin{array}{c}3.4 \pm 0.97 \\
(0.92 \pm 0.17)\end{array}$ & $\begin{array}{c}3.36 \pm 0.74 \\
(0.82 \pm 0.18)\end{array}$ \\
\hline \multirow[t]{4}{*}{ M1 10s } & Bogota & $9.86 \pm 2.1$ & $\begin{array}{r}11.62 \pm 3.79 \\
(1.1 \pm 0.52)\end{array}$ & $\begin{array}{c}15.28 \pm 5.95 \\
(1.46 \pm 0.59)\end{array}$ & $\begin{array}{l}12.99 \pm 4.62 \\
(1.02 \pm 0.54)\end{array}$ & $\begin{array}{r}13.23 \pm 3.99 \\
(1.1 \pm 0.27)\end{array}$ & $\begin{array}{l}11.06 \pm 3.1 \\
(1.12 \pm 0.31)\end{array}$ \\
\hline & $-50 \mathrm{Hgmm}$ & $10.46 \pm 4.87$ & $\begin{array}{c}10.56 \pm 4.37 \\
(1.24 \pm 0.66)\end{array}$ & $\begin{array}{c}11.9 \pm 5.18 \\
(1.52 \pm 1.2)\end{array}$ & $\begin{array}{c}9.58 \pm 6.08 \\
(0.97 \pm 0.86)\end{array}$ & $\begin{array}{c}11.11 \pm 5.59 \\
(1.19 \pm 0.92)\end{array}$ & $\begin{array}{c}10.18 \pm 6.76 \\
(0.96 \pm 0.65)\end{array}$ \\
\hline & -100 Hgmm & $9.28 \pm 4.7$ & $\begin{array}{c}10.78 \pm 5.11 \\
(0.78 \pm 0.32)\end{array}$ & $\begin{array}{c}10.56 \pm 5.07 \\
(0.93 \pm 0.33)\end{array}$ & $\begin{array}{l}11.99 \pm 6.58 \\
(1.38 \pm 0.99)\end{array}$ & $\begin{array}{c}11.37 \pm 6.49 \\
(0.93 \pm 0.63)\end{array}$ & $\begin{array}{c}10.01 \pm 5.53 \\
(0.88 \pm 1.02)\end{array}$ \\
\hline & $-150 \mathrm{Hgmm}$ & $9.1 \pm 3.17$ & $\begin{array}{c}9.73 \pm 4.42 \\
(1.03 \pm 0.34)\end{array}$ & $\begin{array}{c}12.27 \pm 4.79 \\
(1.19 \pm 0.19)\end{array}$ & $\begin{aligned} 12.85 & \pm 4.78 \\
(1.2 & \pm 0.26)\end{aligned}$ & $\begin{array}{r}10.42 \pm 5.61 \\
(1 \pm 0.43)\end{array}$ & $\begin{array}{r}8.4 \pm 3.43 \\
(1.04 \pm 0.5)\end{array}$ \\
\hline \multirow[t]{4}{*}{$\mathrm{AI}[\%]$} & Bogota & $71.31 \pm 4.21$ & $\begin{array}{l}73.53 \pm 3.37 \\
(1.03 \pm 0.03)\end{array}$ & $\begin{array}{c}73.31 \pm 4.48 \\
(1.028 \pm 0.04)\end{array}$ & $\begin{array}{c}71.52 \pm 4.38 \\
(1.003 \pm 0.04)\end{array}$ & $\begin{array}{c}68.39 \pm 3.22^{\S} \\
(0.97 \pm 0.06)\end{array}$ & $\begin{array}{c}67.59 \pm 2.73^{* \S} \\
(0.96 \pm 0.4)\end{array}$ \\
\hline & $-50 \mathrm{Hgmm}$ & $70.19 \pm 5.58$ & $\begin{aligned} 68.97 & \pm 15.68 \\
(0.97 & \pm 0.23)\end{aligned}$ & $\begin{array}{c}72.58 \pm 18.97 \\
(1.05 \pm 0.25)\end{array}$ & $\begin{array}{c}71.73 \pm 5.31 \\
(1.01 \pm 0.08)\end{array}$ & $\begin{array}{c}66.16 \pm 3.65 \\
(0.94 \pm 0.08)\end{array}$ & $\begin{array}{l}67.15 \pm 4^{*} \delta \\
(0.95 \pm 0.07)\end{array}$ \\
\hline & -100 Hgmm & $68.81 \pm 6.01$ & $\begin{array}{l}72.18 \pm 3.19 \\
(1.05 \pm 0.09)\end{array}$ & $\begin{array}{c}67.21 \pm 8.02 \\
(0.97 \pm 0.06)\end{array}$ & $\begin{array}{c}70.63 \pm 5.62 \\
(1.01 \pm 0.06)\end{array}$ & $\begin{array}{c}62.97 \pm 7.95^{*} \\
(0.89 \pm 0.08)\end{array}$ & $\begin{array}{c}65.45 \pm 5.97 \\
(0.93 \pm 0.07)\end{array}$ \\
\hline & $-150 \mathrm{Hgmm}$ & $70.35 \pm 4.28$ & $\begin{array}{c}70.89 \pm 3.17 \\
(1.01 \pm 0.06)\end{array}$ & $\begin{array}{l}70.14 \pm 3.9 \\
(0.99 \pm 0.06)\end{array}$ & $\begin{array}{c}71.53 \pm 3.16 \\
(1.02 \pm 0.07)\end{array}$ & $\begin{array}{r}62.3 \pm 6.62^{*} \\
(0.89 \pm 0.06)\end{array}$ & $\begin{array}{r}63.3 \pm 2.76^{*} \\
(0.92 \pm 0.03)\end{array}$ \\
\hline \multirow[t]{2}{*}{ Amp } & Bogota & $19.41 \pm 2.51$ & $\begin{array}{c}19.18 \pm 2.04 \\
(0.99 \pm 0.13)\end{array}$ & $\begin{array}{c}16.98 \pm 4.07 \\
(0.87 \pm 0.17)\end{array}$ & $\begin{array}{c}16.96 \pm 3.41 \\
(0.87 \pm 0.16)\end{array}$ & $\begin{array}{c}17.84 \pm 2.96 \\
(0.94 \pm 0.2)\end{array}$ & $\begin{array}{c}15.46 \pm 3.75 \\
(0.83 \pm 0.31)\end{array}$ \\
\hline & $-50 \mathrm{Hgmm}$ & $12.28 \pm 5.24$ & $\begin{array}{l}12.49 \pm 6.13 \\
(1.31 \pm 1.14)\end{array}$ & $\begin{array}{c}12.91 \pm 6.92 \\
(1.18 \pm 0.51)\end{array}$ & $\begin{array}{r}11.52 \pm 6.71 \\
(1.3 \pm 0.87)\end{array}$ & $\begin{array}{c}19.69 \pm 2.41 \\
(1.77 \pm 0.92)\end{array}$ & $\begin{array}{c}17.23 \pm 1.23 \\
(0.57 \pm 0.9)\end{array}$ \\
\hline
\end{tabular}


Table 1

(Continued)

\begin{tabular}{|c|c|c|c|c|c|c|c|}
\hline \multirow[t]{2}{*}{ Variable } & \multirow[t]{2}{*}{ Group } & \multirow[t]{2}{*}{ Base } & \multicolumn{3}{|c|}{ Intra-abdominal hypertension phase (3 hours) } & \multicolumn{2}{|c|}{ Treatment phase ( 2 hours) } \\
\hline & & & $1 \mathrm{~h}$ & $2 \mathrm{~h}$ & $3 \mathrm{~h}$ & $4 \mathrm{~h}$ & $5 \mathrm{~h}$ \\
\hline \multirow{6}{*}{$\mathrm{t}_{1 / 2}[\mathrm{~s}]$} & $-100 \mathrm{Hgmm}$ & $16.16 \pm 3.74$ & $\begin{array}{c}15.85 \pm 2.39 \\
(1.08 \pm 0.49)\end{array}$ & $\begin{array}{c}16.04 \pm 3.84 \\
(1.09 \pm 0.51)\end{array}$ & $\begin{array}{c}14.78 \pm 1.91 \\
(1.01 \pm 0.3)\end{array}$ & $\begin{array}{c}19.6 \pm 3.47 \\
(1.4 \pm 0.66)\end{array}$ & $\begin{array}{c}17.2 \pm 3.65 \\
(1.12 \pm 0.12)\end{array}$ \\
\hline & $-150 \mathrm{Hgmm}$ & $17.18 \pm 2.44$ & $\begin{array}{c}16.48 \pm 4.57 \\
(0.95 \pm 0.24)\end{array}$ & $\begin{array}{r}18.75 \pm 4.32 \\
(1.1 \pm 0.27)\end{array}$ & $\begin{array}{c}15.38 \pm 6.18 \\
(0.94 \pm 0.48)\end{array}$ & $\begin{array}{l}16.52 \pm 6.23 \\
(1.01 \pm 0.48)\end{array}$ & $\begin{array}{c}18.72 \pm 2.72 \\
(1.11 \pm 0.32)\end{array}$ \\
\hline & Bogota & $1.42 \pm 0.33$ & $\begin{array}{c}1.23 \pm 0.27 \\
(0.87 \pm 0.11)\end{array}$ & $\begin{array}{c}1.26 \pm 0.38 \\
(0.88 \pm 0.11)\end{array}$ & $\begin{array}{c}1.36 \pm 0.36 \\
(0.96 \pm 0.14)\end{array}$ & $\begin{array}{c}1.62 \pm 0.28^{+\S} \\
(1.11 \pm 0.26)\end{array}$ & $\begin{array}{r}1.69 \pm 0.28^{\S} \\
(1.14 \pm 0.16)\end{array}$ \\
\hline & $-50 \mathrm{Hgmm}$ & $1.57 \pm 0.41$ & $\begin{array}{c}1.4 \pm 0.45 \\
(0.9 \pm 0.28)\end{array}$ & $\begin{array}{c}1.57 \pm 1.06 \\
(0.91 \pm 0.42)\end{array}$ & $\begin{array}{c}1.25 \pm 0.68 \\
(0.97 \pm 0.22)\end{array}$ & $\begin{array}{c}1.77 \pm 0.28 \\
(1.19 \pm 0.23)\end{array}$ & $\begin{array}{c}1.83 \pm 0.41 \\
(1.21 \pm 0.18)\end{array}$ \\
\hline & $-100 \mathrm{Hgmm}$ & $1.71 \pm 0.5$ & $\begin{array}{c}1.33 \pm 0.31 \\
(0.83 \pm 0.25)\end{array}$ & $\begin{array}{c}1.89 \pm 0.86 \\
(1.09 \pm 0.23)\end{array}$ & $\begin{array}{c}1.56 \pm 0.5 \\
(0.99 \pm 0.2)\end{array}$ & $\begin{array}{c}2.32 \pm 1.07 \\
(1.45 \pm 0.36)\end{array}$ & $\begin{array}{c}2.02 \pm 0.68 \\
(1.29 \pm 0.31)\end{array}$ \\
\hline & $-150 \mathrm{Hgmm}$ & $1.5 \pm 0.28$ & $\begin{array}{c}1.38 \pm 0.2 \\
(0.94 \pm 0.18)\end{array}$ & $\begin{array}{c}1.47 \pm 0.23 \\
(1.01 \pm 0.27)\end{array}$ & $\begin{array}{c}1.36 \pm 0.25 \\
(0.93 \pm 0.22)\end{array}$ & $\begin{array}{c}2.31 \pm 0.83^{*} \\
(1.43 \pm 0.33)\end{array}$ & $\begin{array}{c}2.09 \pm 0.3^{*} \\
(1.32 \pm 0.15)\end{array}$ \\
\hline
\end{tabular}

means \pm S.D., and relative values vs. base in parenthesis. ${ }^{*} p<0.05$ vs. Base (within the same group), ${ }^{+} p<0.05 \mathrm{vs} .-100 \mathrm{mmHg}$, $\S_{p}<0.05$ vs. $-150 \mathrm{mmHg}$ (ANOVA test, Bonferroni/Dunn methods).

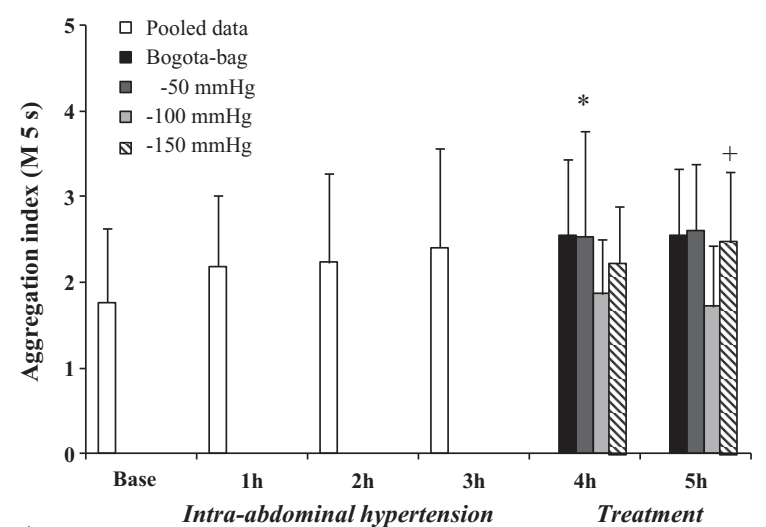

A

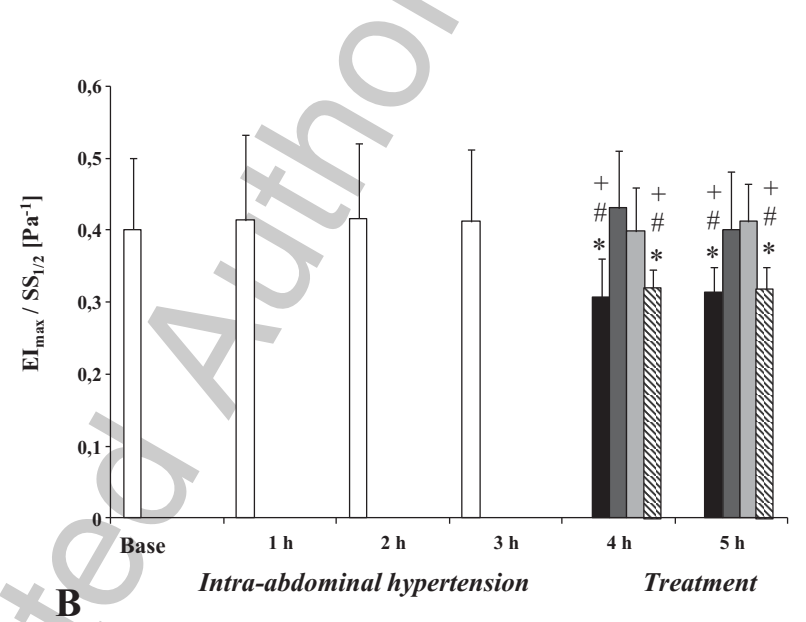

Fig. 1. Red blood cell aggregation index (M $5 \mathrm{~s}$ ) (A) and deformability describing $\mathrm{EI}_{\max } / \mathrm{SS}_{1 / 2}\left[\mathrm{~Pa}^{-1}\right]$ values (B) during the 3-hour 30-mmHg intra-abdominal hypertension period, and after decompression, during the consecutive 2-hour application of various abdominal closure techniques ("Treatment": Bogota-bag, NPWT at $-50,-100$ and $-150 \mathrm{mmHg}$ ). means \pm S.D., ${ }^{*} p<0.05$ vs. Base (within the same group), ${ }^{\#} p<0.05$ vs. $-50 \mathrm{mmHg},{ }^{+} p<0.05 \mathrm{vs} .-100 \mathrm{mmHg}$ (ANOVA test, Bonferroni/Dunn methods).

end of IAH $130.05 \pm 5.93$, end of experiment: $130 \pm 5.81$ ). Maximal EI values also showed slight but non-significant decrease (I. Bogota-group base: $0.532 \pm 0.011$, end of IAH: $0.525 \pm 0.018$, end of experiment $0.525 \pm 0.013$; II. $-50 \mathrm{mmHg}$ group base: $0.531 \pm 0.009$, end of IAH: $0.519 \pm 0.022$, end of experiment: $0.524 \pm 0.013$; III. $-100 \mathrm{mmHg}$ group base: $0.533 \pm 0.013$, end of IAH: $0.522 \pm 0.006$, end of experiment: $0.521 \pm 0.005$; IV. $-150 \mathrm{mmHg}$ group base: $0.531 \pm 0.014$, end of IAH $0.521 \pm 0.017$, end of experiment: $0.519 \pm 0.017)$.

\subsection{Microcirculation of intraabdominal organs}

The results of the laser Doppler microcirculatory test showed obvious differences between the organs not only in the values but the in characteristics of the Laser Doppler signal (amplitude, curve, shape). 
In case of liver (Fig. 2A), the highest microcirculatory blood flux units were recorded in the $-100 \mathrm{mmHg}$ group, while Bogota-group and $-150 \mathrm{mmHg}$ group expressed lower BFU values. On kidney records (Fig. 2B) the decrease compared to base values were well-visible in the Bogota and the $-150 \mathrm{mmHg}$ groups. In the -50 and $-100 \mathrm{mmHg}$ groups we did not observe important decrease in BFU. In data of the pancreas (Fig. 2C) there was no important difference compared to the base, probably due to the anatomical position of the organ. Concerning the small intestine data (Fig. 2D) the worst values were seen in the Bogota-group, and the highest BFU data were expressed in the $-100 \mathrm{mmHg}$ group. Data obtained from the greater omentum decreased in all groups (Fig. 2E), since its position changed during the procedure and it underwent direct compression as well.

\section{Discussion}

Abdominal compartment syndrome is defined as a disease with very high mortality rate, the treatment of which is still a big challenge even nowadays $[13,24]$. If the conservative therapy has failed, surgery is required $[6,25]$. Although there are non-operative possibilities for the treatment of IAH and ACS, the definitive management involves decompressive laparotomy in order to decrease the pressure and temporary closure of the abdominal wall until the disease exists. Decompressive laparotomy may

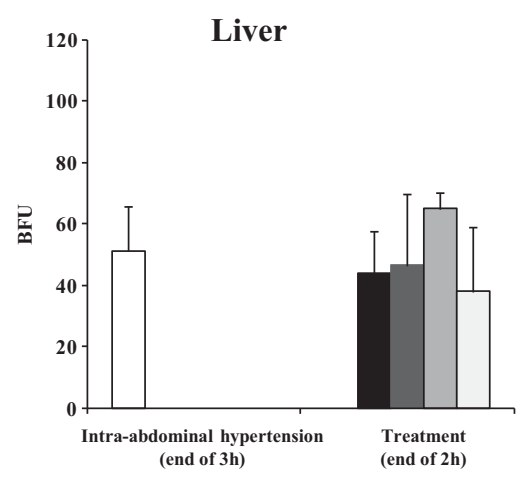

A

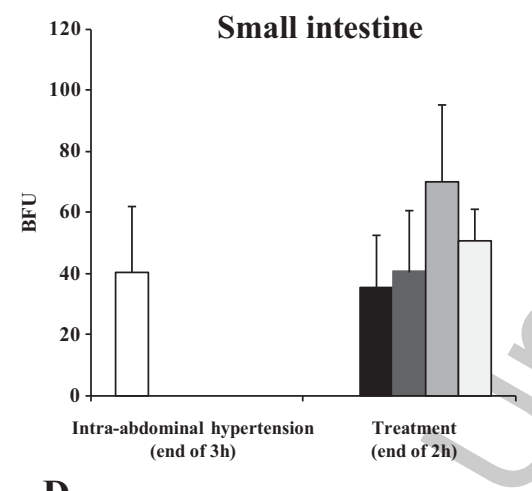

D

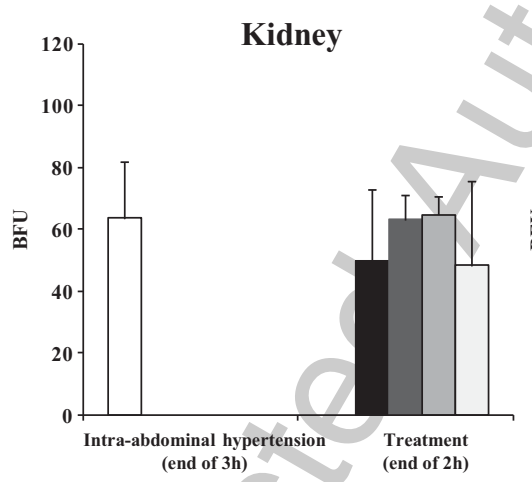

B

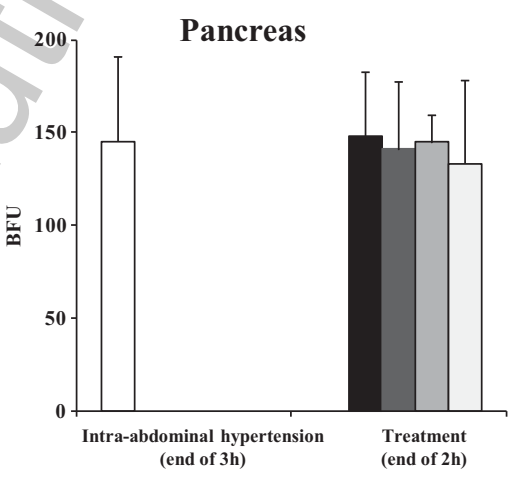

C

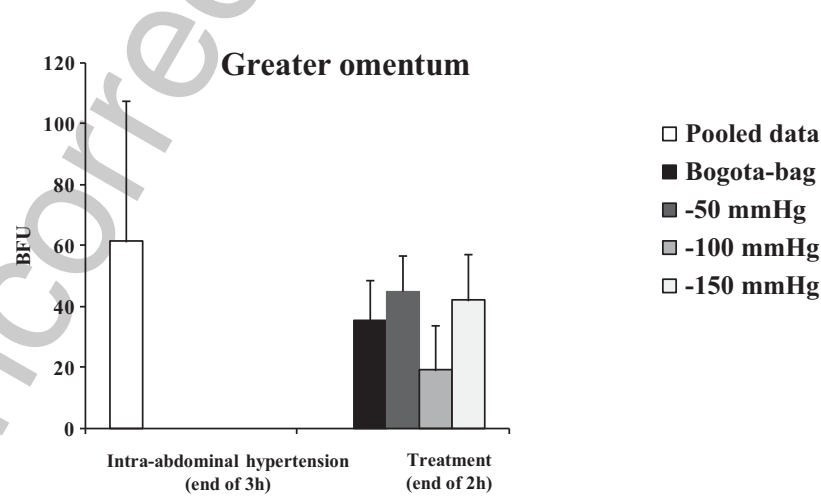

$\mathbf{E}$

Fig. 2. Mean microcirculatory blood flux units (BFU) recorded on the surface of the liver (A), right kidney (B), pancreas (C), jejunum (D) and the greater omentum (E) at the end of the 3-hour 30-mmHg intra-abdominal hypertension period, and after the decompression and the consecutive 2-hour application of various abdominal closure techniques ("Treatment": Bogota-bag, NPWT at $-50,-100$ and $-150 \mathrm{mmHg}$ ). means \pm S.D. 
occur as a life-saving intervention and is followed usually by temporary closure of the abdominal wall $[6,8,11]$. The open abdomen technique aims to protect the abdominal contents, drain intraperitoneal fluids and save the fascia and skin. The generally recommended method is the VAC - negative pressure wound therapy (NPWT) [12, 15, 27]. The consensus guideline of the World Society of the Abdominal Compartment Syndrome in 2013 recommended NPWT in case of critically ill patients $[15,16]$. Although NPWT proved to be favorable in many aspects, but there are very few, objectively justified data about the optimal value of the vacuum in the literature.

In case of increased abdominal pressure, local and systemic disturbances in the circulation may occur, accompanied by deterioration of the hemorheological parameters. We have assumed that measuring the microcirculation of the abdominal organs and analysis of the hemorheological parameters (red blood cell deformability, red blood cell aggregation, whole blood and plasma viscosity) influencing microcirculation, may help to find the optimal negative pressure value.

Our investigation showed that experimental abdominal compartment syndrome resulted in deterioration of micro-rheological parameters, especially in case of RBCs aggregation. NPWT with $-50 \mathrm{mmHg}$ and $-100 \mathrm{mmHg}$ settings led to better results in blood viscosity, RBCs aggregation and RBCs deformability than the use of Bogota bag or NPWT with $-150 \mathrm{mmHg}$.

It can be assumed that the extent of the local circulatory disturbances might have had an impact on RBCs aggregation and RBCs deformability mostly via local metabolic effects [3]. Observing the serosal surface of the small bowel with direct contact to the foil of the vacuum set, imprints of the foil pattern and petechiae were visible even macroscopically at the end of the 2-hour "treatment" period. It was most pronounced in $-150 \mathrm{mmHg}$ group. That also should have contributed to micro-rheological changes.

Increased intra-abdominal pressure is accompanied by decreased microcirculation of the intraabdominal organs, as high pressure in a confined space decreases circulation. It leads to organ hypoperfusion that results in ischemia and ultimately leads to severe organ dysfunction. Furthermore, ischemia-reperfusion injury may cause further harms during decompression of the abdominal cavity. The microcirculatory parameters of Bogota bag and $-150 \mathrm{mmHg}$ NPWT group proved to be bad compared to the values of the -50 and $-100 \mathrm{mmHg}$ group. It could be observed in varying extent by the different organs.

However, the method has some limitations you have to consider during the evaluation. Laser Doppler flowmetry results can be influenced by several factors, such as drying/cooling or movement of the tissue (breathing, movement or trembling). Temperature, instability of the device, tightness of the optic fiber and too close contact with the tissue might all affect the actual value [17]. All these factors have been tried to minimize during the measurements.

\section{Conclusion}

The abdominal compartment syndrome results in deteriorating micro-rheological parameters. Furthermore, the Bogota-bag and the $-150 \mathrm{mmHg}$ negative pressure wound therapy (NPWT) have given worse microcirculatory and micro-rheological results than the -50 or the $-100 \mathrm{mmHg}$ adjustments. These data may contribute to the optimization of NPWT in the surgical management of abdominal compartment syndrome.

\section{Acknowledgments}

The authors are grateful for the technical staff of the Department of Operative Techniques and Surgical Research, Faculty of Medicine, University of Debrecen. 
The authors comply with the Ethical Guidelines for Publication in Clinical Hemorheology and Microcirculation as published on the IOS Press website and in Volume 63, 2016, pp. 1-2. of this journal.

\section{References}

[1] G.M. Arabadzhiev, V.G. Tzaneva and K.G. Peeva, Intra-abdominal hypertension in the ICU-A prospective epidemiological study, Clujul Med 88(2) (2015), 188-195.

[2] O.K. Baskurt, M.R. Hardeman, M. Uyuklu, P. Ulker, M. Cengiz, N. Nemeth, et al., Parameterization of red blood cell elongation index-shear stress curves obtained by ektacytometry, Scand J Clin Lab Invest 69(7) (2009), 777-788.

[3] O.K. Baskurt, Mechanisms of blood rheology alterations. In: Baskurt OK, Hardeman HR, Rampling MW, Meiselman HJ, editors. Handbook of Hemorheology and Hemodynamics. Amsterdam: IOS Press; 2007, pp. 170-190.

[4] E. Benninger, M.W. Laschke, M. Cardell, M. Keel, B. Seifert, O. Trentz, et al., Intra-abdominal pressure development after different temporary abdominal closure techniques in a porcine model, J Trauma 66(4) (2009), 1118-1124.

[5] W.L. Biffl, E.E. Moore, J.M. Burch, P.J. Offner, R.J. Franciose and J.L. Johnson, Secondary abdominal compartment syndrome is a highly lethal event, Am J Surg 182(6) (2001), 645-648.

[6] O. Chiara, S. Cimbanassi, S. Boati and G. Bassi, Surgical management of abdominal compartment syndrome, Minerva Anestesiol 77(4) (2011), 457-462.

[7] G.R. Cokelet and H.J. Meiselman, Macro- and micro-rheological properties of blood. In: Baskurt OK, Hardeman HR, Rampling MW, Meiselman HJ, editors. Handbook of Hemorheology and Hemodynamics. Amsterdam: IOS Press, 2007, pp. 242-266.

[8] J.J. De Waele and A.K. Leppäniemi, Temporary abdominal closure techniques, Am Surg 77(Suppl) (2011), S46-S50.

[9] M.R. Hardeman, P.T. Goedhart and S. Shin, Methods in hemorheology. In: Baskurt OK, Hardeman HR, Rampling MW, Meiselman HJ, editors. Handbook of Hemorheology and Hemodynamics. Amsterdam: IOS Press, 2007, pp. 242-266.

[10] P. Hayden, Intra abdominal hypertension and the abdominal compartment syndrome, Current Anaesthesia \& Critical Care 18 (2007), 311-316.

[11] A. Hecker, B. Hecker, M. Hecker, J.G. Riedel, M.A. Weigand and W. Padberg, Acute abdominal compartment syndrome: Current diagnostic and therapeutic options, Langenbecks Arch Surg 401(1) (2016), 15-24.

[12] H.T. Hougaard, M. Ellebaek, U.T. Holst and N. Qvist, The open abdomen: Temporary closure with a modified negative pressure therapy technique, Int Wound J 11(Suppl 1) (2014), 13-16.

[13] D. Iyer, P. Rastogi, A. Åneman and S. D’Amours, Early screening to identify patients at risk of developing intra-abdominal hypertension and abdominal compartment syndrome, Acta Anaesthesiol Scand 58(10) (2014), 1267-1275.

[14] F. Jung, C. Mrowietz, B. Hiebl, R.P. Franke, G. Pindur and R. Sternitzky, Influence of rheological parameters on the velocity of erythrocytes passing nailfold capillaries in humans, Clin Hemorheol Microcirc 48(1) (2011), $129-139$.

[15] A.W. Kirkpatrick, D.J. Roberts, J. De Waele, R. Jaeschke, M.L. Malbrain, B. De Keulenaer, et al., Intra-abdominal hypertension and the abdominal compartment syndrome: Updated consensus definitions and clinical practice guidelines from the World Society of the Abdominal Compartment Syndrome, Intensive Care Med 39(7) (2013), 1190-1206.

[16] A.W. Kirkpatrick, D.J. Roberts, R. Jaeschke, J.J. De Waele, B.L. De Keulenaer, J. Duchesne, et al., Methodological background and strategy for the 2012-2013 updated consensus definitions and clinical practice guidelines from the abdominal compartment society, Anaesthesiol Intensive Ther 47 (2015), Spec No:s63-77.

[17] M.J. Leahy, Microcirculation Imaging. Hoboken: Wiley-Blackwell; 2012.

[18] R.K. Lee, Intra-abdominal hypertension and abdominal compartment syndrome: A comprehensive overview, Crit Care Nurse 32(1) (2012), 19-31.

[19] H.H. Lipowsky, Microvascular rheology and hemodynamics, Microcirculation 12(1) (2005), 5-15.

[20] L. Maddison, K.M. Riigor, J. Karjagin and J. Starkopf, Sublingual microcirculatory changes during transient intraabdominal hypertension - a prospective observational study in laparoscopic surgery patients, Clin Hemorheol Microcirc 57(4) (2014), 367-374.

[21] M.L. Malbrain, B.L. De Keulenaer, J. Oda, I. De Laet, J.J. De Waele, D.J. Roberts, et al., Intra-abdominal hypertension and abdominal compartment syndrome in burns, obesity, pregnancy, and general medicine, Anaesthesiol Intensive Ther 47(3) (2015), 228-240.

[22] M.L. Malbrain, I.E. De Laet, J.J. De Waele and A.W. Kirkpatrick, Intra-abdominal hypertension: Definitions, monitoring, interpretation and management, Best Pract Res Clin Anaesthesiol 27(2) (2013), 249-270.

[23] A. Matrai, R.B. Whittington and E. Ernst, A simple method of estimating whole blood viscosity at standardized hematocrit, Clin Hemorheol 7 (1987), 261-265. 
[24] D.J.J. Muckart, R.R. Ivatury, A. Leppaniemi and R.S. Smith, Definitions. In: Ivatury RR, Cheatham ML, Malbrain M, Sugrue M, editors. Abdominal Compartment Syndrome. Georgetown: Landis Bioscience; 2006, pp. 8-18.

[25] P. Rastogi, D. Iyer, A. Aneman and S. D' Amours, Intra-abdominal hypertension and abdominal compartment syndrome: Pathophysiological and non-operative management, Minerva Anestesiol 80(8) (2014), 922-932.

[26] D.J. Roberts, C.G. Ball and A.W. Kirkpatrick, Increased pressure within the abdominal compartment: Intra-abdominal hypertension and the abdominal compartment syndrome, Curr Opin Crit Care 22(2) (2016), 174-185.

[27] A. Surace, A. Ferrarese, S. Marola, J. Cumbo, G. Valentina, A. Borello, et al., Abdominal compartment syndrome and open abdomen management with negative pressure devices, Ann Ital Chir 86(1) (2015), 46-50.

[28] Z. Szentkereszty and A. Csiszko, Abdominal compartment syndrome in severe acute pancreatitis - literature review, Magy Seb 65(1) (2012), 9-13. 